\title{
Characteristics Associated to Lipodystrophy Syndrome among HIV- Infected Patients Naive and on Antiretroviral Treatment
}

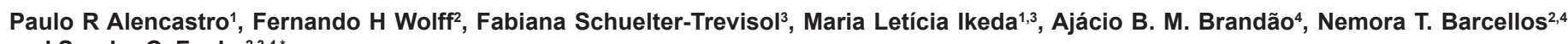
and Sandra C. Fuchs $\mathbf{s}^{2,3,4, *}$

${ }^{1}$ Hospital Sanatório Partenon, Rio Grande do Sul State Department of Health, Av. Bento Gonçalves, 3722, Porto Alegre, RS 90650-001, Brazil ${ }^{2}$ Postgraduate Program in Epidemiology, School of Medicine. Universidade Federal do Rio Grande do Sul, R. Ramiro Barcelos 2600, Porto Alegre, RS 90035-003, Brazil ${ }^{3}$ Postgraduate Program in Cardiology, School of Medicine, Universidade Federal do Rio Grande do Sul, R. Ramiro Barcelos 2350, Porto Alegre, RS 90035-003, Brazil ${ }^{4}$ National Institute for Health Technology Assessment (IATS/CNPq), Hospital de Clinicas de Porto Alegre, Centro de Pesquisa Clínica, R. Ramiro Barcelos 2350, Porto Alegre, RS 90035-003, Brazil

\begin{abstract}
Background: HIV-associated lipodystrophy involves changes in complex metabolic networks that are associated with increased cardiovascular risk. It has been associated with the use of combined antiretroviral treatment (cART), particularly Protease Inhibitors $(\mathrm{PI})$ and thymidine analogs. This study aims to evaluate characteristics and use of ART associated with lipodystrophy, lipohypertrophy, and lipoatrophy among HIV-infected patients.

Methods: A cross-sectional study was conducted in HIV-infected patients of both genders, aged 18 years or older, who sought care at an HIVIAIDS referral service for diagnostic confirmation or treatment between June 2006 and December 2008.

Results: 1240 out of 1295 patients with HIV infection were included. Among patients on cART, women had a higher risk of lipohypertrophy than men, as well as a time since diagnosis of HIV greater than 6 years (versus $<3$ years). For lipoatrophy, age, education, lifestyle, and body mass index were associated with increased risk Metabolic parameters were higher among patients on ART; and CART and PI use were independently associated with lipohypertrophy, lipoatrophy and lipodystrophy. The use of PI can be regarded as responsible for $13 \%$ of the association of ART and lipodystrophy, and of $11.5 \%$ for the thymidine analogs use, independent of gender, skin color, smoking, CD4, and BMI.
\end{abstract}

Conclusions: Risk factors for lipodystrophy, lipoatrophy and lipohypertrophy are marked among ART users, but also among ART naïve patients.

Keywords: HIV; Lipodystrophy; Lipohypertrophy; Lipoatrophy; Risk factors; HAART; Dyslipidemia

\section{Introduction}

The introduction of combined antiretroviral treatment (cART) of HIV was a milestone in the struggle to reduce the rate of morbidity associated with progression of disease toward advanced stages of immunosuppression [1]. HIV-infected patients with lipodystrophy exhibit clinical changes in complex metabolic networks that are associated with increased cardiovascular risk [2-6].

Lipodystrophy is characterized by dyslipidemia, visceral adiposity, and subcutaneous abdominal fat buildup with peripheral wasting. The features of fat redistribution are variable, but they are usually detected by accumulation of fat in the abdomen, chest, breasts, or dorsocervical fat pad ("buffalo hump"), signs of lipohypertrophy, and the wasting of the face, anterior and lateral region of the neck, legs, arms or buttocks, known as lipoatrophy $[3,5,7,8]$. The HIV-associated lipodystrophy is a multifactorial disorder due to the interaction between virus and host factors related to cART [9]. Lipodystrophy appears to be mediated, even before the start of cART, by the increased inflammatory cytokines resulting from the HIV infection itself and, later, by use of cART [6]. The Protease Inhibitor (PI) reduces the proliferation and differentiation of adipocytes and increases lipolysis by inhibition of CRABP-1 (cytoplasmic retinoic-acid binding protein type1), blocking the activation of transcription factors linked to the PPAR- $\gamma$ (peroxizone proliferator activated receptor type gamma) [10]. Nucleoside Reverse Transcriptase Inhibitor (especially stavudine-D4T) induce mitochondrial dysfunction [11], leading to lipoatrophy $[6,9,10]$. The prevalence of lipodystrophy ranges from 18 to $83 \%$, depending on the criteria used for diagnosis [12] and along with body fat changes, there are metabolic abnormalities including increased levels of triglycerides, LDL cholesterol, total cholesterol, glucose, and insulin, and decreased level of HDL cholesterol [3,5].

There are few data available on ART among HIV-infected people living in low and middle income countries [13,14]. In Senegal, thymidine analogs and PI are among the most commonly used drugs for the treatment of HIV-infected patients, which contributes to the prevalence of lipodystrophy 65\% [14]. In another African study, conducted in Rwanda, the prevalence of lipodystrophy was $34 \%$, and the patients were not in treatment with PI [13]. Few studies conducted in Brazil have assessed the prevalence and risk factors of lipodystrophy in HIVinfected patients who are not participating of randomized clinical trials [15]. In a study conducted in São Paulo, lipodystrophy was detected in

*Corresponding author: Sandra C. Fuchs, Instituto de Avaliação de Tecnologias em Saúde (IATS) Centro de Pesquisa Clínica, $5^{\circ}$ andar Hospital de Clínicas de Porto Alegre, Universidade Federal do Rio Grande do Sul, Rua Ramiro Barcellos, 2350, Porto Alegre, RS, 90035-003, Brazil, Tel: +55 51 3359-7621/3359-8420 E-mail: scfuchs@terra.com.br

Received September 03, 2012; Accepted November 12, 2012; Published November 22, 2012

Citation: Alencastro PR, Wolff FH, Schuelter-Trevisol F, Ikeda ML, et al. (2012) Characteristics Associated to Lipodystrophy Syndrome among HIV-Infected Patients Naïve and on Antiretroviral Treatment. J AIDS Clinic Res 3:182. doi:10.4172/21556113.1000182

Copyright: (C) 2012 Alencastro PR, et al. This is an open-access article distributed under the terms of the Creative Commons Attribution License, which permits unrestricted use, distribution, and reproduction in any medium, provided the original author and source are credited. 
Citation: Alencastro PR, Wolff FH, Schuelter-Trevisol F, Ikeda ML, et al. (2012) Characteristics Associated to Lipodystrophy Syndrome among HIVInfected Patients Naïve and on Antiretroviral Treatment. J AIDS Clinic Res 3:182. doi:10.4172/2155-6113.1000182

64.3\% of patients, $37.4 \%$ had lipoatrophy and lipohypertrophy $49.2 \%$ [16]. In this study we aimed to verify characteristics associated to lipodystrophy syndrome among HIV-infected patients naïve and on ART.

\section{Methods}

This cross-sectional study included men and women living with HIV/AIDS, aged 18 years or older, who sought HIV diagnostic confirmation or treatment at the HIV/AIDS outpatient care center of Hospital Sanatório Partenon. Pregnant women, patients with intellectual disability, and incarcerated or institutionalized persons were excluded from the study. The study was approved by the institutional review board of the Hospital de Clínicas de Porto Alegre, which is accredited by the Office of Human Research Protections. All participants signed a consent form.

\section{Study variables}

We used a standardized questionnaire to collect data on demographic (age, gender, skin color), socioeconomic (years at school), lifestyle characteristics (smoking and physical activity), HIV-infection related variables: use of antiretroviral treatment (ART), time since HIV diagnosis (categorized as $<3,3-5$, and $\geq 6$ ), and signs and symptoms of lipodystrophy. Age was calculated by subtracting the birth date from the date of interview; with analysis conducted as a categorical variable (18-34, 35-49, and 50-78 years) for the description and as a continuous variable to detect independent associations; skin color was self-reported and categorized as Caucasian or non-Caucasian; and education was measured as the number of years at school, analyzed as a categorical variable $(0-4,5-8,9-11$, or $\geq 12$ years $)$ in the description and as a continuous variable to assess independent associations. Lifetime tobacco exposure was calculated in former and current smokers by the number of packs smoked per day $(1$ pack $=20$ cigarettes $)$ multiplied by the number of years of smoking [17-19]. For purposes of analysis, former or current smokers were stratified into those with $\geq 20$ and those with $<20$ pack-years. Physical activity was estimated by the IPAQ (International Physical Activity Questionnaire) instrument [20], and subjects were considered physically active if they engaged weekly in at least 150 minutes of physical activity $[21,22]$. Body mass index (BMI, $\mathrm{kg} / \mathrm{m}^{2}$ ) was assessed as a categorical variable for the description and a continuous variable in order to detect the independent associations. The use of ART was investigated during anamnesis and confirmed with the recorded information in the clinical center for use of any drug during lifetime, protease inhibitors (PI), and cART, which was defined as the use of three or more drugs over the 12 months preceding the study. Patients who have never been exposed to ART were classified as ART naïve. The time of diagnosis of HIV was reported by the patient and confirmed through a review of medical records and the dates of HIV testing.

A 12 hours fasting glucose, lipid profile, CD4-lymphocyte counts and HIV RNA viral load were requested for patients who had not been tested in the last three months. Laboratory tests were performed using standard techniques [23-25]. Dyslipidemia was detected by total cholesterol $\geq 200 \mathrm{md} / \mathrm{dL}$ or HDL cholesterol $<40 \mathrm{mg} / \mathrm{dL}$ [26] and use of lipid-lowering treatment was further investigated.

\section{Diagnosis of lipodystrophy}

Lipodystrophy was identified by objective measurements and by the report of increased or decreased body fat. Objective measures were determined by measurement of waist circumference, hip, arm and neck; and skin folds in infra-orbital, buccal and submandibular regions with anthropometric caliper. The $10^{\text {th }}$ and $90^{\text {th }}$ percentiles were used to define the cutoffs for lipoatrophy and lipohypertrophy, respectively [27]. The change in body fat located in face, chin, back of neck, chest or breasts, abdomen, arms, forearms and hands, hips and buttocks, thighs, legs and feet could be determined by the perception of muscular arms or legs, prominent superficial veins, presence of a "buffalo hump", abdominal enlargement, double chin, hollow cheeks, or changes in the width of the neck or waist. Finally, lipohypertrophy and lipoatrophy were established by the presence of at least two self-perceived signs or objective measurements of hypertrophy or atrophy, respectively $[12,28]$, while those who had one of these conditions were classified as having lipodystrophy.

\section{Data collection}

Data were collected during routine visits, and consisted of blood pressure measurement and anthropometry. Weight $(\mathrm{kg})$ and height $(\mathrm{m})$ were measured, with patients in barefoot and wearing light clothes. Body mass index (BMI) was calculated by dividing the weight (in $\mathrm{kg}$ ) by the height (in meters) squared. Waist circumference was measured midway between the iliac crest and low costal rib margin [29,30]. Hip circumference was measured at the greater trochanter and the point of greatest gluteal protuberance.

Facial skin fold thickness was measured with a scientific skin fold caliper in the infra-orbital, buccal and submandibular regions. Measurements were done in duplicate and the average was used for determine the abnormal cutoffs.

\section{Sample size calculation and statistical analysis}

Sample size calculation was based on an estimate that $17 \%$ of ART naïve patients and $25 \%$ of patients on ART would have lipodystrophy. For a statistical power of $80 \%$ and $95 \%$ confidence interval, with an unexposed to exposed ratio of 0.5 , in order to detect a risk ratio (RR) of 1.5, it was needed to enroll at least 984 patients. The sample size was increased in $30 \%$ to take into account confounding factors in multivariate analysis.

Pearson's chi-squared test was used for categorical variables and analysis of variance, for continuous variables. All analyses were performed in the Statistical Package for the Social Sciences (SPSS) version 14.0 software environment (SPSS Inc., Chicago, Illinois, USA). Modified Poisson regression was used to test for associations between risk factors and the presence of lipohypertrophy, lipoatrophy, and lipodystrophy, calculating adjusted prevalence ratios and $95 \%$ confidence intervals. A trend toward association was defined as $0.05<\mathrm{P}<0.1$.

The analysis was based on a hierarchical framework, which provides a strategy to conduct multivariate analysis in studies where determinants of disease are sought, there are hierarchical relationships among determinants, and these aspects are taken into consideration as well as the literature [29]. The information allowed to testing some characteristics as continuous and categorical variables, and the best fit was used in each statistical test. Characteristics were aggregated into three sets of variables, which contained different hierarchical levels of determination, including in the first level: socioeconomic (education) and biological characteristics (gender, age, and skin color), in the second: lifestyle (smoking and physical activity), and in the third level: HIV-related characteristics (viral load, CD4, time since HIV diagnosis, and BMI). These potential determinants are likely to lead to lipohypertrophy, lipoatrophy, and lipodystrophy (Figure 1). At each level, variables were included in the model based on the strength 
Citation: Alencastro PR, Wolff FH, Schuelter-Trevisol F, Ikeda ML, et al. (2012) Characteristics Associated to Lipodystrophy Syndrome among HIVInfected Patients Naïve and on Antiretroviral Treatment. J AIDS Clinic Res 3:182. doi:10.4172/2155-6113.1000182

Page 3 of 9

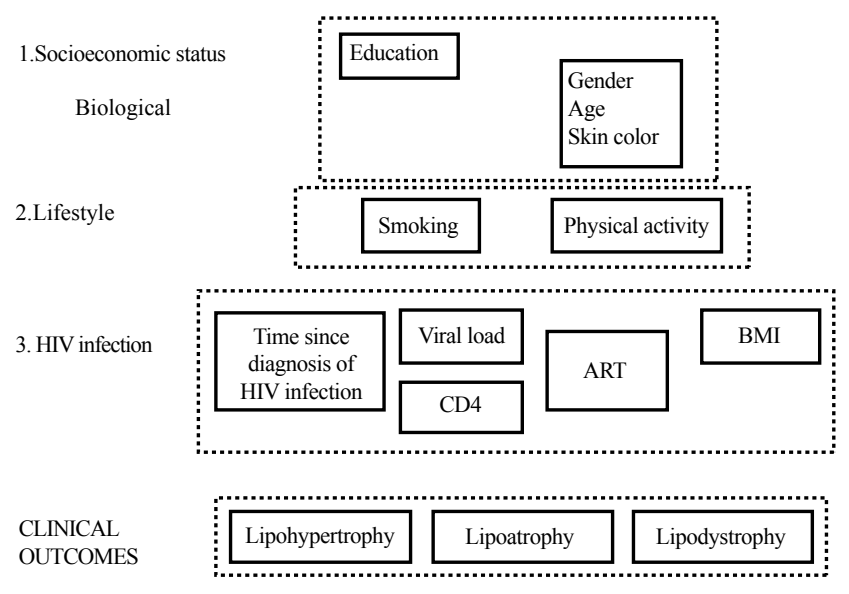

Figure 1: Levels of hierarchical model.

of association in the crude analyses ( $\mathrm{P}$ value $<0.2)$, and one regression equation was fitted for each hierarchical level, also including variables from higher levels of determination [30]. We conducted separate analyses according to the ART status (on ART and ART naïve) and for each clinical outcome.

\section{Results}

The cohort included a consecutive sample of 1240 , out of 1295 , HIV-infected subjects eligible to participate, 15 refused to take part, and 40 were excluded due to age, incarceration, or pregnancy. Participants were, on average, $38.6 \pm 10.1$ years old. Approximately half were men, most were Caucasians. Most patients had received ART (65.7\%) during lifetime, and among those on ART the majority used PI (42.6\%), thymidine analogs (31.0\%), and d-drugs (31.9\%).

Table 1 describes characteristics of patients receiving ART and ART naïve subjects. Patients on cART were older $(\mathrm{P}<0.001)$, smokers $(\mathrm{P}=0.004)$, had increased $\mathrm{BMI}(\mathrm{P}=0.003)$, longer time since HIV infection diagnosis $(\mathrm{P}<0.001)$, had detectable viral load $(\mathrm{P}<0.001)$, CD 4 counts below 350 cells $/ \mathrm{mm}^{3}(\mathrm{P}<0.001)$, and metabolic parameters were higher than those patients who were cART naïve. There were 51 patients on ART and six, who had not started treatment at the time of the study enrollment, with advanced stages of AIDS.

The prevalence of lipohypertrophy was $50.2 \%$ among patients on ART and $37.9 \%$ among ART naïve patients, $58.2 \%$ and $43.8 \%$, respectively, for lipoatrophy, and $79.1 \%$ and $64.7 \%$, respectively, for lipodystrophy. Table 2 presents the distribution of characteristics associated with lipohypertrophy, lipoatrophy, and lipodystrophy separated for patients on CART and ART naïve.

Table 3 shows the characteristics independently associated with risk of lipohypertrophy, lipoatrophy and lipodystrophy in patients on ART. The selection of confounding factors for lipohypertrophy were: gender and age (first level), smoking (second level), and BMI, CD4, and viral load (third level); for lipoatrophy: gender, skin color, and education (first level), none for the second level, and BMI and time since diagnosis of HIV (third level); for lipodystrophy: age, skin color, and education (first level), none for the second level, and BMI and time since diagnosis of HIV (third level). Women had 58\% higher risk of lipohypertrophy than men $(\mathrm{P}<0.001)$, independent of age. After control for age and gender, smoking status remained as a protective factor for lipohypertrophy while an increase in one unit of BMI resulted in
7\% elevated risk of lipohypertrophy, after the control for biological, lifestyle, and HIV-related variables. Time since HIV diagnosis was not associated with lipohypertrophy. After the control for gender, skin color, and educations, characteristics as non-white skin color increased $26 \%$ the risk of lipoatrophy, in comparison to white ones $(\mathrm{P}<0.001)$, while education was inversely associated $(\mathrm{P}<0.001)$. Body mass index was inverse and independently associated to lipoatrophy $(\mathrm{P}<0.001)$ and longer time since the HIV diagnosis was a risk factor. Lipodystrophy

\begin{tabular}{|c|c|c|c|c|}
\hline & $\begin{array}{l}\text { Overall } \\
(n=1240)\end{array}$ & $\begin{array}{l}\text { ART } \\
(n=815)\end{array}$ & $\begin{array}{l}\text { No ART } \\
(n=425)\end{array}$ & P-value \\
\hline Age (years) & $39.1 \pm 10.1$ & $40.5 \pm 9.7$ & $36.5 \pm 10.2$ & $<0.001$ \\
\hline Gender & & & & 0.4 \\
\hline Male & $628(50.6)$ & $420(51.5)$ & $208(48.9)$ & \\
\hline Female & $612(49.4)$ & $395(48.5)$ & $217(51.1)$ & \\
\hline Education (years) & $7.5 \pm 4.1$ & $7.4 \pm 4.1$ & $7.7 \pm 4.1$ & 0.2 \\
\hline Skin color & & & & 0.9 \\
\hline Caucasian & $548(44.2)$ & $348(42.7)$ & $182(42.8)$ & \\
\hline Non-Caucasian & $692(55.8)$ & $467(57.3)$ & $243(57.2)$ & \\
\hline Smoking (pack-years) & & & & 0.004 \\
\hline 0 & $422(34.0)$ & $272(33.4)$ & $150(35.3)$ & \\
\hline $1-19$ & $498(40.2)$ & $309(37.9)$ & $189(44.5)$ & \\
\hline$\geq 20$ & $320(25.8)$ & $234(28.7)$ & $86(20.2)$ & \\
\hline $\begin{array}{l}\text { Physical activity (min/ } \\
\text { week) }\end{array}$ & & & & 0.4 \\
\hline$\geq 150$ & $524(42.3)$ & $352(43.2)$ & $172(40.5)$ & \\
\hline$<150$ & $716(57.7)$ & $463(56.8)$ & $253(59.5)$ & \\
\hline $\begin{array}{l}\text { Body mass index }(\mathrm{kg} / \\
\left.\mathrm{m}^{2}\right)\end{array}$ & & & & 0.01 \\
\hline$<18.5$ & $38(3.1)$ & $27(3.3)$ & $11(2.6)$ & \\
\hline $18.5-24.9$ & $670(54.0)$ & $466(57.2)$ & $204(48.0)$ & \\
\hline $25.0-29.9$ & $380(30.6)$ & $232(28.5)$ & $148(34.8)$ & \\
\hline$\geq 30.0$ & $152(12.3)$ & $90(11.0)$ & $62(14.6)$ & \\
\hline $\begin{array}{l}\text { Time since diagnosis of } \\
\text { HIV infection (years) }\end{array}$ & & & & $<0.001$ \\
\hline$<3$ & 485 (39.1) & $211(25.9)$ & $274(64.5)$ & \\
\hline $3-5$ & $350(28.2)$ & $256(31.4)$ & $94(22.1)$ & \\
\hline$\geq 6$ & $405(32.7)$ & $347(42.6)$ & $58(13.4)$ & \\
\hline Viral load (copies/mL) & & & & $<0.001$ \\
\hline$<50$ & $713(58.4)$ & $321(39.7)$ & $392(95.1)$ & \\
\hline$\geq 50$ & $508(41.6)$ & $488(60.3)$ & $20(4.9)$ & \\
\hline CD4 (cells/mm³) & & & & $<0.001$ \\
\hline$\geq 350$ & $751(61.2)$ & $451(55.6)$ & $300(72.1)$ & \\
\hline $200-349$ & $295(24.0)$ & $215(26.5)$ & $80(19.2)$ & \\
\hline$<200$ & $181(14.8)$ & $145(17.9)$ & $36(8.7)$ & \\
\hline Glucose (mg/dL) & $86.8 \pm 27.6$ & $88.2 \pm 29.9$ & $84.3 \pm 22.3$ & 0.02 \\
\hline Triglycerides $(\mathrm{mg} / \mathrm{dL})$ & $151.9 \pm 107.5$ & $167.9 \pm 121.1$ & $120.6 \pm 63.3$ & $<0.001$ \\
\hline Total cholesterol (mg/dL) & $183.1 \pm 43.6$ & $187.9 \pm 45.7$ & $173.7 \pm 37.5$ & $<0.001$ \\
\hline HDL cholesterol (mg/dL) & $51.2 \pm 13.8$ & $51.9 \pm 14.1$ & $49.8 \pm 13.1$ & 0.01 \\
\hline LDL cholesterol (mg/dL) & $103.0 \pm 35.8$ & $104.4 \pm 37.4$ & $100.4 \pm 32.4$ & 0.07 \\
\hline \multicolumn{5}{|l|}{ Dyslipidemia* } \\
\hline Yes & 608 (49.6) & $431(53.3)$ & $177(42.5)$ & $<0.001$ \\
\hline No & $617(50.4)$ & $378(46.7)$ & $239(57.5)$ & \\
\hline \multicolumn{5}{|l|}{$\begin{array}{l}\text { Use of lipid-lowering } \\
\text { treatment }\end{array}$} \\
\hline Yes & $29(2.3)$ & $26(3.2)$ & $3(0.7)$ & 0.006 \\
\hline No & $1211(97.7)$ & $789(96.8)$ & $422(99.3)$ & \\
\hline
\end{tabular}

* Dyslipidemia: total cholesterol $\geq 200 \mathrm{md} / \mathrm{dL}$ or HDL cholesterol $<40 \mathrm{mg} / \mathrm{dL}$

Table 1: Characteristics of HIV-infected patients, stratified by ART use [n(\%) or mean \pm SD]. 
Citation: Alencastro PR, Wolff FH, Schuelter-Trevisol F, Ikeda ML, et al. (2012) Characteristics Associated to Lipodystrophy Syndrome among HIVInfected Patients Naïve and on Antiretroviral Treatment. J AIDS Clinic Res 3:182. doi:10.4172/2155-6113.1000182

Page 4 of 9

\begin{tabular}{|c|c|c|c|c|c|c|}
\hline & \multicolumn{3}{|c|}{ On ART } & \multicolumn{3}{|c|}{ ART naïve } \\
\hline & Lipohypertrophy & Lipoatrophy & Lipodystrophy & Lipohypertrophy & Lipoatrophy & Lipodystrophy \\
\hline \multicolumn{7}{|l|}{ Age (years) } \\
\hline $18-34$ & $127(51.2)$ & $136(54.8)$ & $187(75.4)$ & $84(37.7)$ & $104(46.6)$ & $147(65.9)$ \\
\hline $35-49$ & $208(47.5)$ & $265(60.5)$ & $349(79.7)$ & $58(37.9)$ & $64(41.8)$ & 99 (64.7) \\
\hline $50-78$ & $74(57.4)$ & $73(56.6)$ & $109(84.5)$ & $19(38.8)$ & $18(36.7)$ & $29(59.2)$ \\
\hline$P$ value & 0.13 & 0.3 & 0.11 & 1.0 & 0.4 & 0.7 \\
\hline \multicolumn{7}{|l|}{ Gender } \\
\hline Male & $167(39.8)$ & $248(59.0)$ & $327(77.9)$ & $75(36.1)$ & $90(43.3)$ & $138(66.3)$ \\
\hline Female & $242(61.3)$ & $226(57.2)$ & $318(80.5)$ & 86 (39.6) & $96(44.2)$ & $137(63.1)$ \\
\hline$P$ value & $<0.001$ & 0.6 & 0.4 & 0.4 & 0.8 & 0.5 \\
\hline \multicolumn{7}{|l|}{ Education (years) } \\
\hline $0-4$ & $116(53.0)$ & $136(62.1)$ & $183(83.6)$ & $28(27.5)$ & $56(54.9)$ & $66(64.7)$ \\
\hline $5-8$ & $151(49.2)$ & $199(64.8)$ & $252(82.1)$ & 71 (44.9) & $74(46.8)$ & $114(72.2)$ \\
\hline $9-11$ & $96(49.0)$ & $101(51.5)$ & $145(74.0)$ & $42(36.5)$ & $40(34.8)$ & $67(58.3)$ \\
\hline$\geq 12$ & 46 (49.5) & 38 (40.9) & 65 (69.9) & $20(40.0)$ & $16(32.0)$ & $28(56.0)$ \\
\hline$P$ value & 0.8 & $<0.001$ & 0.007 & 0.04 & 0.006 & 0.06 \\
\hline \multicolumn{7}{|l|}{ Skin color } \\
\hline Caucasian & $185(53.2)$ & $234(67.2)$ & $297(85.3)$ & 78 (42.9) & $87(47.8)$ & $127(69.8)$ \\
\hline Non-Caucasian & $224(48.0)$ & $240(51.4)$ & $348(74.5)$ & $83(34.2)$ & 99 (40.7) & $148(60.9)$ \\
\hline $\mathrm{P}$ value & 0.14 & $<0.001$ & $<0.001$ & 0.07 & 0.15 & 0.06 \\
\hline \multicolumn{7}{|c|}{ Smoking (pack-years) } \\
\hline 0 & $160(58.8)$ & $150(55.1)$ & $215(79.0)$ & $61(40.7)$ & $52(34.7)$ & 89 (59.3) \\
\hline $1-19$ & $144(46.6)$ & $182(58.9)$ & $242(78.3)$ & $67(35.4)$ & 83 (43.9) & $120(63.5)$ \\
\hline$\geq 20$ & $105(44.9)$ & $142(60.7)$ & $188(80.3)$ & $33(38.4)$ & $51(59.3)$ & $66(76.7)$ \\
\hline$P$ value & 0.002 & 0.4 & 0.8 & 0.6 & $<0.001$ & 0.02 \\
\hline \multicolumn{7}{|c|}{ Physical activity (min/week) } \\
\hline$\geq 150$ & $179(50.9)$ & $276(59.6)$ & 367 (79.3) & $94(37.2)$ & $121(47.8)$ & $168(66.4)$ \\
\hline$<150$ & $230(49.7)$ & $198(56.3)$ & $278(79.0)$ & $67(39.0)$ & $65(37.8)$ & $107(62.2)$ \\
\hline$P$ value & 0.7 & 0.3 & 0.9 & 0.7 & 0.04 & 0.4 \\
\hline \multicolumn{7}{|c|}{ Body mass index $\left(\mathrm{kg} / \mathrm{m}^{2}\right)$} \\
\hline$<18.5$ & $3(11.1)$ & $27(100.0)$ & $27(100.0)$ & $1(9.1)$ & $11(100.0)$ & $11(100.0)$ \\
\hline $18.5-24.9$ & $170(36.5)$ & $298(63.9)$ & 355 (76.2) & $49(24.0)$ & 109 (53.4) & $134(65.7)$ \\
\hline $25-29.9$ & $156(67.2)$ & $104(44.8)$ & $181(78.0)$ & $64(43.2)$ & $48(32.4)$ & $82(55.4)$ \\
\hline$\geq 30.0$ & $80(88.9)$ & $45(50.0)$ & $82(91.1)$ & $47(75.8)$ & $18(29.0)$ & $48(77.4)$ \\
\hline$P$ value & $<0.001$ & $<0.001$ & 0.001 & $<0.001$ & $<0.001$ & 0.001 \\
\hline \multicolumn{7}{|c|}{$\begin{array}{l}\text { Time since diagnosis of HIV } \\
\text { infection (years) }\end{array}$} \\
\hline$<3$ & $108(51.2)$ & $113(53.6)$ & $160(75.8)$ & $98(35.8)$ & $121(44.2)$ & $170(62.0)$ \\
\hline $3-5$ & $128(50.0)$ & $138(53.9)$ & $196(76.6)$ & $41(43.6)$ & $36(38.3)$ & $63(67.0)$ \\
\hline$\geq 6$ & $173(49.9)$ & $223(64.3)$ & $289(83.3)$ & $22(38.6)$ & $29(50.9)$ & $42(73.7)$ \\
\hline$P$ value & 1.0 & 0.01 & 0.048 & 0.4 & 0.3 & 0.2 \\
\hline \multicolumn{7}{|c|}{ Viral load (copies/mL) } \\
\hline$<50$ & $272(55.7)$ & $270(55.3)$ & $390(79.7)$ & $8(40.0)$ & $9(45.0)$ & $11(55.0)$ \\
\hline$\geq 50$ & $134(41.7)$ & $200(62.3)$ & $250(77.9)$ & $148(37.8)$ & $169(43.1)$ & $255(65.1)$ \\
\hline$P$ value & $<0.001$ & 0.049 & 0.5 & 0.8 & 0.9 & 0.4 \\
\hline \multicolumn{7}{|l|}{ CD4 (cells/mm³) } \\
\hline$\geq 350$ & $242(53.7)$ & $256(56.8)$ & 356 (78.9) & $130(43.3)$ & $131(43.7)$ & $197(65.7)$ \\
\hline $200-349$ & $114(53.0)$ & $126(58.6)$ & $175(81.4)$ & $21(26.3)$ & $30(37.5)$ & $48(60.0)$ \\
\hline$<200$ & $51(35.2)$ & $90(62.1)$ & $111(76.6)$ & $7(19.4)$ & $19(52.8)$ & $24(66.7)$ \\
\hline$P$ value & $<0.001$ & 0.5 & 0.5 & 0.001 & 0.3 & 0.6 \\
\hline \multicolumn{7}{|l|}{ Dyslipidemia* } \\
\hline Yes & $226(52.4)$ & $239(55.5)$ & $339(78.7)$ & $68(38.4)$ & 76 (42.9) & $117(66.1)$ \\
\hline No & $181(47.9)$ & $230(60.8)$ & $300(79.4)$ & $91(38.1)$ & $105(43.9)$ & $152(63.6)$ \\
\hline$P$ value & 0.2 & 0.12 & 0.8 & 0.9 & 0.8 & 0.6 \\
\hline \multicolumn{7}{|c|}{ Use of lipid-lowering treatment } \\
\hline Yes & $15(57.7)$ & $16(61.5)$ & $22(84.6)$ & 1 (33.3) & $2(66.7)$ & $2(66.7)$ \\
\hline No & $394(49.9)$ & $458(58.0)$ & $623(79.0)$ & $160(37.9)$ & $184(43.6)$ & $273(64.7)$ \\
\hline$P$ value & 0.4 & 0.7 & 0.5 & 0.9 & 0.4 & 0.9 \\
\hline
\end{tabular}

Table 2: Distribution of characteristics associated with lipohypertrophy, lipoatrophy, and lipodystrophy in HIV-infected patients according to ART treatment status [n (\%)]. 
Citation: Alencastro PR, Wolff FH, Schuelter-Trevisol F, Ikeda ML, et al. (2012) Characteristics Associated to Lipodystrophy Syndrome among HIVInfected Patients Naïve and on Antiretroviral Treatment. J AIDS Clinic Res 3:182. doi:10.4172/2155-6113.1000182

Page 5 of 9

\begin{tabular}{|c|c|c|c|}
\hline & Lipohypertrophy & Lipoatrophy & Lipodystrophy \\
\hline & Model $1^{*}$ & Model $1^{f}$ & Model $1^{\ddagger}$ \\
\hline \multicolumn{4}{|l|}{ Gender } \\
\hline Male & 1.00 & 1.00 & 1.00 \\
\hline Female & $1.58(1.37-1.82)$ & $0.92(0.82-1.03)$ & $1.02(0.95-1.10)$ \\
\hline$P$ value & $<0.001$ & 0.16 & 0.5 \\
\hline Age (years) & $1.01(1.00-1.01)$ & $1.00(1.00-1.01)$ & $1.004(1.00-1.01)$ \\
\hline$P$ value & 0.06 & 0.9 & 0.04 \\
\hline \multicolumn{4}{|l|}{ Skin color } \\
\hline Caucasian & 1.00 & 1.00 & 1.00 \\
\hline Non - Caucasian & $1.07(0.94-1.23)$ & $1.26(1.12-1.41)$ & $1.13(1.05-1.21)$ \\
\hline$P$ value & 0.3 & $<0.001$ & $<0.001$ \\
\hline Education (years) & $1.01(0.99-1.02)$ & $0.97(0.96-0.99)$ & $0.99(0.98-0.99)$ \\
\hline \multirow[t]{2}{*}{$P$ value } & 0.4 & $<0.001$ & 0.03 \\
\hline & Model $2^{* *}$ & Model $2^{f}$ & Model $2^{\ddagger}$ \\
\hline \multicolumn{4}{|c|}{ Smoking (pack-years) } \\
\hline 0 & 1.00 & 1.00 & 1.00 \\
\hline $1-19$ & $0.81(0.68-0.97)$ & $1.06(0.92-1.21)$ & $1.00(0.92-1.08)$ \\
\hline$\geq 20$ & $0.81(0.70-0.95)$ & $1.07(0.93-1.25)$ & $0.99(0.91-1.09)$ \\
\hline$P$ value & 0.01 & 0.6 & 1.0 \\
\hline \multicolumn{4}{|l|}{$\begin{array}{l}\text { Physical activity } \\
\text { (min/week) }\end{array}$} \\
\hline$\geq 150$ & 1.00 & 1.00 & 1.00 \\
\hline$<150$ & $1.05(0.91-1.20)$ & $0.96(0.86-1.08)$ & $1.01(0.94-1.08)$ \\
\hline \multirow[t]{2}{*}{$\mathrm{P}$ value } & 0.5 & 0.5 & 0.9 \\
\hline & Model $3^{* * *}$ & Model $3^{f f}$ & Model $3^{\ddagger \ddagger}$ \\
\hline $\begin{array}{l}\text { Body mass index } \\
\left(\mathrm{kg} / \mathrm{m}^{2}\right)\end{array}$ & $1.07(1.06-1.09)$ & $0.96(0.95-0.98)$ & $1.007(1.00-1.01)$ \\
\hline P-value & $<0.001$ & $<0.001$ & 0.04 \\
\hline \multicolumn{4}{|l|}{ CD4 (cells $/ \mathrm{mm}^{3}$ ) } \\
\hline$\geq 350$ & 1.00 & 1.00 & 1.00 \\
\hline $200-349$ & $1.11(0.96-1.28)$ & $0.99(0.87-1.13)$ & $1.02(0.95-1.11)$ \\
\hline$<200$ & $0.83(0.67-1.05)$ & $0.98(0.85-1.13)$ & $0.95(0.86-1.05)$ \\
\hline$P$ value & 0.049 & 1.0 & 0.4 \\
\hline \multicolumn{4}{|c|}{ Viral load (copies $/ \mathrm{mL}$ ) } \\
\hline$<50$ & 1.00 & 1.00 & 1.00 \\
\hline$\geq 50$ & $0.78(0.67-0.91)$ & $1.04(0.93-1.16)$ & $0.96(0.89-1.04)$ \\
\hline$P$ value & 0.001 & 0.5 & 0.3 \\
\hline \multicolumn{4}{|c|}{$\begin{array}{l}\text { Time since diagnosis of } \\
\text { HIV infection (years) }\end{array}$} \\
\hline$<3$ & 1.00 & 1.00 & 1.00 \\
\hline $3-5$ & $1.02(0.87-1.22)$ & $0.97(0.82-1.14)$ & $1.01(0.91-1.12)$ \\
\hline$\geq 6$ & $1.11(0.95-1.29)$ & $1.18(1.02-1.37)$ & $1.12(1.02-1.22)$ \\
\hline$P$ value & 0.4 & 0.005 & 0.01 \\
\hline
\end{tabular}

* Adjusted for gender and age

** Adjusted for gender, age, and smoking status

${ }^{* * *}$ Adjusted for gender, age, smoking status, BMI, CD4, and viral load

${ }^{f}$ Adjusted for gender, skin color and education

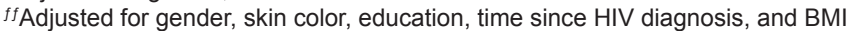

¥Adjusted for age, skin color, and education

¥Adjusted for age, skin color, education, time since HIV diagnosis, and BMI

Table 3: Risk factors for lipodystrophy in HIV - infected patients on antiretroviral treatment (risk ratio and $95 \% \mathrm{Cl}$ ).

was markedly affected by biological characteristics, which increased the risk, except by education that was inversely associated. HIVrelated characteristics-BMI $(\mathrm{P}=0.04)$ and time since the HIV diagnosis $(\mathrm{P}=0.01)$ maintained positive associations with lipodystrophy, even after the control for confounding factors.

Table 4 shows the risk factors for lipodystrophy in patients ART naive, following the same models of control for confounding factors.

The selection of confounding factors for lipohypertrophy were: skin color and education (first level), none for the second level, and BMI and CD4 (third level); for lipoatrophy: age and education (first level), smoking and physical activity (second level), and BMI (third level); for lipodystrophy: skin color and education (first level), smoking (second level), and none for the third level. Non-Caucasian participants were at increased risk of lipohypertrophy (RR 1.31 (95\% CI 1.02-1.68); P=0.03),

\begin{tabular}{|c|c|c|c|}
\hline & Lipohypertrophy & Lipoatrophy & Lipodystrophy \\
\hline & Model $1^{*}$ & Model $1^{\dagger}$ & Model $1^{*}$ \\
\hline \multicolumn{4}{|l|}{ Gender } \\
\hline Male & 1.00 & 1.00 & 1.00 \\
\hline Female & $1.09(0.85-1.40)$ & $0.97(0.79-1.21)$ & $0.92(0.80-1.06)$ \\
\hline$P$ value & 0.5 & 0.8 & 0.3 \\
\hline Age (years) & $1.00(0.99-1.01)$ & $0.99(0.98-0.99)$ & $1.00(0.99-1.00$ \\
\hline$P$ value & 0.8 & 0.035 & 0.2 \\
\hline \multicolumn{4}{|l|}{ Skin color } \\
\hline Caucasian & 1.00 & 1.00 & 1.00 \\
\hline Non-Caucasian & $1.31(1.02-1.68)$ & $1.09(0.88-1.35)$ & $1.12(0.97-1.29)$ \\
\hline$P$ value & 0.03 & 0.4 & 0.13 \\
\hline Education (years) & $1.02(0.99-1.05)$ & $0.95(0.93-0.98)$ & $0.99(0.97-1.01)$ \\
\hline \multirow[t]{2}{*}{$\mathrm{P}$ value } & 0.18 & 0.001 & 0.19 \\
\hline & Model $2^{*}$ & Model $2^{\text {t† }}$ & Model $2^{\ddagger}$ \\
\hline \multicolumn{4}{|l|}{ Smoking (pack-years) } \\
\hline 0 & 1.00 & 1.00 & 1.00 \\
\hline $1-19$ & $0.86(0.67-1.17)$ & $1.16(0.89-1.52)$ & $1.27(1.07-1.52)$ \\
\hline$\geq 20$ & $0.99(0.71-1.39)$ & $1.75(1.31-2.35)$ & $1.06(0.89-1.26)$ \\
\hline$P$ value & 0.6 & $<0.001$ & 0.016 \\
\hline \multicolumn{4}{|l|}{$\begin{array}{l}\text { Physical activity ( } \mathrm{min} / \\
\text { week) }\end{array}$} \\
\hline$\geq 150$ & 1.00 & 1.00 & 1.00 \\
\hline$<150$ & $1.06(0.83-1.36)$ & $0.80(0.64-1.00)$ & $0.94(0.81-1.08)$ \\
\hline \multirow[t]{2}{*}{$\mathrm{P}$ value } & 0.6 & 0.046 & 0.4 \\
\hline & Model 3** & Model $3^{\text {ttt }}$ & Model $3^{\ddagger}$ \\
\hline Body mass index $\left(\mathrm{kg} / \mathrm{m}^{2}\right)$ & $1.09(1.07-1.11)$ & $0.94(0.91-0.97)$ & $\begin{array}{l}1.00(0.99- \\
1.02)\end{array}$ \\
\hline$P$ value & $<0.001$ & $<0.001$ & 0.6 \\
\hline \multicolumn{4}{|l|}{ CD4 (cells $/ \mathrm{mm}^{3}$ ) } \\
\hline$\geq 350$ & 1.00 & 1.00 & 1.00 \\
\hline $200-349$ & $0.71(0.49-1.03)$ & $0.85(0.63-1.16)$ & $0.90(0.74-1.09)$ \\
\hline$<200$ & $0.58(0.30-1.11)$ & $1.16(0.85-1.59)$ & $0.98(0.77-1.26)$ \\
\hline$P$ value & 0.07 & 0.3 & 0.5 \\
\hline \multicolumn{4}{|l|}{ Viral load (copies/mL) } \\
\hline$<50$ & 1.00 & 1.00 & 1.00 \\
\hline$\geq 50$ & $0.98(0.57-1.70)$ & $0.88(0.54-1.42)$ & $1.16(0.77-1.73)$ \\
\hline$P$ value & 1.0 & 0.6 & 0.5 \\
\hline \multicolumn{4}{|l|}{$\begin{array}{l}\text { Time since diagnosis of } \\
\text { HIV infection (years) }\end{array}$} \\
\hline$<3$ & 1.00 & 1.00 & 1.00 \\
\hline 3-5 & $1.12(0.87-1.45)$ & $0.86(0.65-1.15)$ & $1.06(0.90-1.26)$ \\
\hline$\geq 6$ & $1.25(0.88-1.77)$ & $0.96(0.72-1.29)$ & $1.11(0.92-1.34)$ \\
\hline$P$ value & 0.4 & 0.6 & 0.5 \\
\hline
\end{tabular}

*Adjusted for skin color and education

${ }^{* *}$ Adjusted for skin color, education, BMI, and CD4

${ }^{\dagger}$ Adjusted for age and education

${ }^{+\dagger}$ Adjusted for age and education, physical activity, and smoking

${ }^{\mathrm{tt}}$ Adjusted for age and education, physical activity, smoking, and BMI

${ }^{\ddagger}$ Adjusted for skin color, education, and smoking

Table 4: Risk factors for lipodystrophy in HIV- infected patients ART naive (risk ratio and $95 \% \mathrm{Cl}$ ). 
Citation: Alencastro PR, Wolff FH, Schuelter-Trevisol F, Ikeda ML, et al. (2012) Characteristics Associated to Lipodystrophy Syndrome among HIVInfected Patients Naïve and on Antiretroviral Treatment. J AIDS Clinic Res 3:182. doi:10.4172/2155-6113.1000182

Page 6 of 9

\begin{tabular}{|c|c|c|c|c|c|}
\hline & Cholesterol $^{*}$ & HDL cholesterol* & LDL cholesterol* & Triglycerides* & Glucose* \\
\hline \multicolumn{6}{|l|}{ ART } \\
\hline \multicolumn{6}{|c|}{ Lipohypertrophy } \\
\hline No & $179.9 \pm 2.2$ & $51.0 \pm 0.7$ & $100.2 \pm 1.9$ & $150.2 \pm 5.9$ & $86.1 \pm 1.5$ \\
\hline Yes & $195.7 \pm 2.2$ & $52.7 \pm 0.7$ & $108.4 \pm 1.9$ & $185.4 \pm 5.8$ & $90.2 \pm 1.5$ \\
\hline P-value & $<0.001$ & 0.09 & 0.002 & $<0.001$ & 0.05 \\
\hline \multicolumn{6}{|c|}{ Lipoatrophy } \\
\hline No & $195.6 \pm 2.4$ & $52.4 \pm 0.8$ & $108.8 \pm 2.1$ & $175.6 \pm 6.5$ & $89.8 \pm 1.6$ \\
\hline Yes & $182.2 \pm 2.0$ & $51.1 \pm 0.7$ & $101.1 \pm 1.7$ & $162.4 \pm 5.5$ & $87.0 \pm 1.4$ \\
\hline$P$ value & $<0.001$ & 0.09 & 0.005 & 0.1 & 0.2 \\
\hline \multicolumn{6}{|c|}{ Lipodystrophy } \\
\hline No & $188.5 \pm 3.5$ & $52.4 \pm 1.1$ & $104.8 \pm 2.9$ & $162.5 \pm 9.2$ & $86.0 \pm 2.3$ \\
\hline Yes & $187.7 \pm 1.8$ & $51.7 \pm 0.6$ & $104.2 \pm 1.5$ & $169.4 \pm 4.7$ & $88.7 \pm 1.2$ \\
\hline$P$ value & 0.8 & 0.6 & 0.9 & 0.5 & 0.3 \\
\hline \multicolumn{6}{|c|}{ ART naïve } \\
\hline \multicolumn{6}{|c|}{ Lipohypertrophy } \\
\hline No & $169.8 \pm 2.2$ & $49.0 \pm 0.8$ & $98.6 \pm 2.0$ & $112.3 \pm 3.9$ & $81.6 \pm 1.4$ \\
\hline Yes & $180.5 \pm 2.8$ & $51.2 \pm 1.0$ & $103.2 \pm 2.5$ & $134.0 \pm 4.9$ & $88.7 \pm 1.7$ \\
\hline P-value & 0.003 & 0.08 & 0.2 & 0.001 & 0.001 \\
\hline \multicolumn{6}{|c|}{ Lipoatrophy } \\
\hline No & $171.7 \pm 2.4$ & $49.1 \pm 0.9$ & $98.7 \pm 2.1$ & $118.1 \pm 4.1$ & $84.8 \pm 1.5$ \\
\hline Yes & $176.3 \pm 2.7$ & $50.7 \pm 1.0$ & $102.5 \pm 2.4$ & $123.8 \pm 4.7$ & $83.6 \pm 1.7$ \\
\hline P-value & 0.2 & 0.2 & 0.3 & 0.4 & 0.6 \\
\hline \multicolumn{6}{|c|}{ Lipodystrophy } \\
\hline No & $168.9 \pm 3.0$ & $49.3 \pm 1.1$ & $97.0 \pm 2.6$ & $110.5 \pm 5.2$ & $80.9 \pm 1.8$ \\
\hline Yes & $176.3 \pm 1.2$ & $50.1 \pm 0.8$ & $102.2 \pm 2.0$ & $126.1 \pm 3.8$ & $86.2 \pm 1.3$ \\
\hline P-value & 0.047 & 0.6 & 0.1 & 0.02 & 0.02 \\
\hline
\end{tabular}

${ }^{*} \mathrm{mg} / \mathrm{dL}$.

${ }^{* \star}$ Adjusted for age, skin color, smoking status, and time since HIV diagnosis

Table 5: Metabolic profile (mean \pm SEM) of HIV-infected patients with lipodystrophy ${ }^{* *}$

\begin{tabular}{|c|c|c|c|}
\hline & Lipohypertrophy & Lipoatrophy & Lipodystrophy \\
\hline Antiretroviral treatment (ART) & $1.33(1.15-1.52)^{*}$ & $1.33(1.18-1.50)^{\star}$ & $1.22(1.13-1.32)^{\star}$ \\
\hline ART+gender & $1.34(1.16-1.54)^{*}$ & $1.33(1.18-1.50)^{*}$ & $1.22(1.13-1.32)^{*}$ \\
\hline ART+age (years) & $1.31(1.14-1.51)^{*}$ & $1.34(1.18-1.52)^{\star}$ & $1.22(1.12-1.32)^{*}$ \\
\hline ART+skin color & $1.34(1.16-1.54)^{\star}$ & $1.34(1.18-1.51)^{*}$ & $1.23(1.14-1.33)^{*}$ \\
\hline ART, complete model ${ }^{\#}$ & $1.49(1.30-1.70)^{\star}$ & $1.30(1.15-1.47)^{*}$ & $1.23(1.13-1.33)^{*}$ \\
\hline Protease inhibitors (PI) & $1.16(1.03-1.31)^{\star \star}$ & $1.22(1.10-1.36)^{*}$ & $1.14(1.07-1.22)^{*}$ \\
\hline $\mathrm{Pl}+$ gender & $1.13(1.00-1.27)^{\star \star}$ & $1.23(1.11-1.36)^{*}$ & $1.14(1.07-1.22)^{*}$ \\
\hline $\mathrm{PI}+$ age (years) & $1.15(1.02-1.30)^{\Lambda}$ & $1.22(1.10-1.36)^{*}$ & $1.14(1.07-1.21)^{*}$ \\
\hline $\mathrm{Pl}+$ skin color & $1.17(1.04-1.32)^{\star \star}$ & $1.24(1.12-1.38)^{*}$ & $1.15(1.08-1.23)^{*}$ \\
\hline PI, complete model & $1.23(1.10-1.38)^{*}$ & $1.20(1.08-1.32)^{*}$ & $1.15(1.07-1.22)^{*}$ \\
\hline
\end{tabular}

\#Adjusted for gender, skin color, smoking status, BMI and CD4.

${ }^{*} \mathrm{P}$ value $<0.001 ;{ }^{* *} \mathrm{P}$ value $0.01 \leq \mathrm{P} \leq 0.05 ;{ }^{\Delta} \mathrm{P}$ value $=0.19$

Table 6: Modified Poisson regression for risk factors associated with lipohypertrophy, lipoatrophy and lipodystrophy (risk ratio and $95 \% \mathrm{Cl}$ ).

compared with Caucasians, independent of skin color and education. Furthermore, after adjusting for skin color, education, and CD4, BMI was associated with higher risk [RR 1.09 (95\% CI 1.07-1.11); $\mathrm{P}<0.001]$. As for lipoatrophy, age $(\mathrm{P}=0.035)$, education $(\mathrm{P}=0.001)$, and physical activity $(\mathrm{P}=0.046)$ showed an inverse effect on risk, even after the control for confounding factors. Patients who smoked 20 or more packyears had $75 \%$ the risk $(\mathrm{RR}=1.75(95 \% \mathrm{CI} 1.31-2.35)$ in comparison to never smokers. For lipodystrophy, an independent association was detected only with smoking $(\mathrm{P}=0.016)$, but there was no dose-response.

Table 5 describes the metabolic parameters observed in patients on cART and not on cART, according to lipohypertrophy, lipoatrophy and lipodystrophy. All metabolic parameters were adjusted for age, skin color, smoking status, and time since HIV diagnosis. Those on
cART who had lipohypertrophy had higher mean levels of nearly all metabolic parameters. Conversely, those with lipoatrophy had lower mean cholesterol $(\mathrm{P}<0.001)$ and LDL-cholesterol ( $\mathrm{P}$ 0.005) levels. Among ART naive patients, those with lipohypertrophy had higher total cholesterol ( $\mathrm{P}$ 0.003), triglycerides ( $\mathrm{P}$ 0.001), and glucose $(\mathrm{P}$ 0.001 ) levels, while for lipodystrophy the metabolic abnormalities were observed (P 0.047, P 0.02, P 0.02, respectively).

Table 6 shows that use of cART and PI was independently associated with lipohypertrophy, lipoatrophy and lipodystrophy. The use of ART increased 23\%, 30\%, and 49\% the risk of lipodystrophy, lipoatrophy, and lipohypertrophy, respectively, while for PI use they were $15 \%$, $20 \%$, and $23 \%$, respectively, after adjustment for biological, lifestyle, and HIV-related characteristics. Additional analysis showed that 
Citation: Alencastro PR, Wolff FH, Schuelter-Trevisol F, Ikeda ML, et al. (2012) Characteristics Associated to Lipodystrophy Syndrome among HIVInfected Patients Naïve and on Antiretroviral Treatment. J AIDS Clinic Res 3:182. doi:10.4172/2155-6113.1000182

thymidine analogs were associated with higher risk of lipohypertrophy $(\mathrm{RR}=1.47$ (95\%CI: 1.30-1.36), $\mathrm{P}<0.001)$, lipoatrophy $(\mathrm{RR}=1.35$ (95\%CI: 1.22-1.50), $\mathrm{P}<0.001)$, and lipodystrophy ( $\mathrm{RR}=1.27$ (95\%CI:1.20-1.35), $\mathrm{P}<0.001$ ), independent of gender, skin color, smoking, and CD4. In addition, we estimated that use of PI can be regarded as responsible for $13 \%$ of the association of ART and lipodystrophy, and of $11.5 \%$ for the thymidine analogs use, independent of gender, skin color, smoking, CD4, and BMI.

\section{Discussion}

This study showed that in this population several characteristics associated with lipohypertrophy and lipoatrophy phenotypes were different among those on ART and ART naïve. In addition, it also showed that the lipodystrophy category is not so helpful, since risk factors for lipoatrophy and lipohypertrophy are very different. Although lipodystrophy is more frequent in individuals on ART, this study shows that patients ART naïve were also affected [31] and that clinical outcomes can be attributed to the HIV infection.

Lipodystrophy seems to be mediated, even before the institution of cART, by an increase of inflammatory cytokines resulting from the infection itself [3]. Epidemiological studies suggest that cART and factors not related to therapy alike are potential risk factors for body fat redistribution [6,9]. For instance, among ART naive patients, the associations between smoking and lipodystrophy, mainly due to the lipoatrophy component, and the inverse relationship between CD4 levels and lipohipertrophy can be related to the progressive wasting that commonly accompanies the decrease of CD4. The results of our study agree with those verified in a large multicenter, cross-sectional [31]. The multi-factorial explanation of lipodystrophy is corroborated in other reports of body fat, outpatient study carried out in the United States, which showed that lipodystrophy was strongly associated with ART and patient-related factors and that lipohypertrophy was linked to correlates of immunologic recovery. The multi-factorial explanation of lipodystrophy is corroborated in other reports of body fat changes in HIV-1 infected patients who were not on PI [32], but were taken nucleoside reverse transcriptase inhibitors (NRTI) and no PI [31,33].

Few studies have been conducted among HIV-infected patients who were not on ART and most of the known associations derived from studies on patients on cART. This report among patients on ART from Southern Brazil confirmed the risk of lipohypertrophy associated with female gender [3,13,34], increasing age [31,35], high BMI [31], hypertriglyceridemia [34], and abnormal lipid profile $[35,36]$. Regarding lipoatrophy in patients on cART, the results of this study were similar to those described for low BMI $[6,36,37]$, reduced LDL cholesterol, and low total cholesterol. Overall lipodystrophy had confirmed associations with age [35], non-Caucasian skin color, BMI [38], and duration of HIV infection [15]. The comparison of findings among patients on cART and ART naïve allows identifying a common pathway for the association of BMI with lipohypertrophy. Patients with greater fat deposit may slow or mitigate the loss of adipose tissue.

This study also assessed metabolic profile of HIV-infected patients, on ART and ART naïve, mostly associated with metabolic syndrome [39]. A previous multicenter cohort study reported more frequent lipid abnormalities in HIV-infected individuals than in the general population[40]. However, this study showed that lipid levels vary according to the ART status and that the report without taken into account this condition may under detect the prevalence of metabolic abnormalities [41]. A study conducted with patients on cART in Rwanda, showed higher lipid profiles and blood glucose in patients with lipodystrophy than in those who did not have the condition [13]. This study provided further information since lipid and glucose abnormalities were higher in patients with lipohypertrophy and lower among those with lipoatrophy on cART. However, ART naïve patients who had lipohypertrophy also had higher levels of cholesterol, triglyceride and glucose.

The imbalance of metabolic profile has been attributed to insulin resistance [30], which is increased by ART use, but not exclusively [42]. Sensitivity to insulin is worsen among patients on PI treatment who had lipodystrophy [43] and each additional year of exposure to nucleoside analogue reverse transcriptase inhibitors (NRTI) increased $8 \%$ the odds of hyperinsulinemia and $20 \%$ for stavudine [42].

There are some potential limitations that should take into account in the interpretation of the results. The cross-sectional design precludes the establishment of causal relationships and may be affected by potential biases. Ascertainment bias, for example, could have biased the diagnosis of clinical outcomes if the observer knew what ART drugs were in use and their adverse effects. However, the diagnosis was based in a combination of objective measurements or the patients' report of changes in body fat. The data collection was carried out with no information about the hypothesis being tested and what criteria would be used for the diagnosis. However, the study enrolled a large sample size, the data collection was meticulously obtained, all measurements were carried out in duplicate and the average was adopted in the analysis. Finally, the control for confounding factors was based on separated modeling for each clinical outcome and the use of modified Poisson regression was able to provide risk estimates appropriated for the cross-sectional design, what may have mitigated the role of biases. Besides, the simultaneous description of both phenotypes according to the ART status provides information to develop specific interventions to minimize the effect of ART and pathways to investigate causality. The results of this study could be generalized to patients with HIV/AIDS who seek care in the public health system of Porto Alegre, Southern Brazil, and its metropolitan area. Therefore, our patients are alike those from other public health centers, which provide care for most of HIV infected patients.

In conclusion, HIV-associated lipodystrophy occurred in patients with longer duration of HIV infection and on treatment with antiretroviral drugs, in whom metabolic abnormalities, especially dyslipidemia, are frequent and present increased cardiovascular risk. Therefore, these patients should undergo frequent laboratory monitoring and, if indicated, specific treatment should be initiated, since ART use is essential to increase life expectancy and to reduce the burden of disease. However, patients ART naïve were also at increased risk of cardiovascular events due to lipid abnormalities, which could be prevented.

\section{Acknowledgements}

This study was supported by grants and scholarships from the CNPq (National Council for Scientific and Technological Development), CAPES (Coordination for the Improvement of Higher Education Personnel), Fundação de Amparo a Pesquisa do Rio Grande do Sul (FAPERGS), and FIPE-HCPA (Fundo de Apoio a Pesquisa, Hospital de Clínicas de Porto Alegre).

The sponsors did not take part in the design or conduct of the study, including data collection, management, analysis, and interpretation of the data; and preparation, review, or approval of the manuscript.

The authors had full access to all of the data in the study and take responsibility for the integrity of the data and the accuracy of the data analysis.

\section{References}


Citation: Alencastro PR, Wolff FH, Schuelter-Trevisol F, Ikeda ML, et al. (2012) Characteristics Associated to Lipodystrophy Syndrome among HIVInfected Patients Naïve and on Antiretroviral Treatment. J AIDS Clinic Res 3:182. doi:10.4172/2155-6113.1000182

Page 8 of 9

1. Palella FJ Jr, Delaney KM, Moorman AC, Loveless MO, Fuhrer J, et al. (1998) Declining morbidity and mortality among patients with advanced human immunodeficiency virus infection. HIV Outpatient Study Investigators. N Engl J Med 338: 853-860.

2. Hadigan C, Meigs JB, Corcoran C, Rietschel P, Piecuch S, et al. (2001) Metabolic abnormalities and cardiovascular disease risk factors in adults with human immunodeficiency virus infection and lipodystrophy. Clin Infect Dis 32: 130-139.

3. Grinspoon SK (2005) Metabolic syndrome and cardiovascular disease in patients with human immunodeficiency virus. Am J Med 118 Suppl 2: 23S-28S.

4. Dube MP, Stein JH, Aberg JA, Fichtenbaum CJ, Gerber JG, et al. (2003) Guidelines for the evaluation and management of dyslipidemia in human immunodeficiency virus (HIV)-infected adults receiving antiretroviral therapy: recommendations of the HIV Medical Association of the Infectious Disease Society of America and the Adult AIDS Clinical Trials Group. Clin Infect Dis 37: 613-627.

5. ter Hofstede HJ, Burger DM, Koopmans PP (2003) Antiretroviral therapy in HIV patients: aspects of metabolic complications and mitochondrial toxicity. Neth $\mathrm{J}$ Med 61: 393-403.

6. Grinspoon S, Carr A (2005) Cardiovascular risk and body-fat abnormalities in HIV-infected adults. N Engl J Med 352: 48-62.

7. Tien PC, Grunfeld C (2004) What is HIV-associated lipodystrophy? Defining fat distribution changes in HIV infection. Curr Opin Infect Dis 17: 27-32.

8. Domingo P, Estrada V, Lopez-Aldeguer J, Villaroya F, Martinez E (2012) Fat redistribution syndromes associated with HIV-1 infection and combination antiretroviral therapy. AIDS Rev 14:112-123.

9. Leow MK, Addy CL, Mantzoros CS (2003) Clinical review 159: Human immunodeficiency virus/highly active antiretroviral therapy-associated metabolic syndrome: clinical presentation, pathophysiology, and therapeutic strategies. J Clin Endocrinol Metab 88: 1961-1976.

10. Carr A, Samaras K, Chisholm DJ, Cooper DA (1998) Pathogenesis of HIV1-protease inhibitor-associated peripheral lipodystrophy, hyperlipidaemia, and insulin resistance. Lancet 351: 1881-1883.

11. Villarroya F, Domingo P, Giralt M (2005) Lipodystrophy associated with highly active anti-retroviral therapy for HIV infection: the adipocyte as a target of antiretroviral-induced mitochondrial toxicity. Trends Pharmacol Sci 26: 88-93.

12. Carr A (2003) HIV lipodystrophy: risk factors, pathogenesis, diagnosis and management. AIDS 17 Suppl 1: S141-148.

13. Mutimura E, Stewart A, Rheeder P, Crowther NJ (2007) Metabolic function and the prevalence of lipodystrophy in a population of HIV-infected African subjects receiving highly active antiretroviral therapy. J Acquir Immune Defic Syndr 46:451-455.

14. Mercier S, Gueye NF, Cournil A, Fontbonne A, Copin N, et al. (2009) Lipodystrophy and metabolic disorders in HIV-1-infected adults on 4- to 9-year antiretroviral therapy in Senegal: a case-control study. J Acquir Immune Defic Syndr 51: 224-230.

15. Diehl LA, Dias JR, Paes AC, Thomazini MC, Garcia LR, et al. (2008) [Prevalence of HIV-associated lipodystrophy in Brazilian outpatients: relation with metabolic syndrome and cardiovascular risk factors]. Arq Bras Endocrinol Metabol 52: 658-667.

16. Santos CP, Felipe YX, Braga PE, Ramos D, Lima RO, et al. (2005) Selfperception of body changes in persons living with HIVIAIDS: prevalence and associated factors. AIDS 19 Suppl 4: S14-21.

17. Bernaards CM, Twisk JW, Snel J, Van Mechelen W, Kemper HC (2001) Is calculating pack-years retrospectively a valid method to estimate life-time tobacco smoking? A comparison between prospectively calculated pack-years and retrospectively calculated pack-years. Addiction 96: 1653-1661.

18. Halmenschlager G, Rossetto S, Lara GM, Rhoden EL (2009) Evaluation of the effects of cigarette smoking on testosterone levels in adult men. J Sex Med 6 : 1763-1772.

19. Cui Q, Carruthers S, Mclvor A, Smaill F, Thabane L, et al. (2010) Effect of smoking on lung function, respiratory symptoms and respiratory diseases amongst HIV-positive subjects: a cross-sectional study. AIDS Res Ther 7: 6.

20. Craig CL, Marshall AL, Sjöström M, Bauman AE, Booth ML, et al. (2003) International physical activity questionnaire: 12-country reliability and validity.
Med Sci Sports Exerc 35: 1381-1395

21. Pate RR, Pratt M, Blair SN, Haskell WL, Macera CA, et al. (1995) Physical activity and public health. A recommendation from the Centers for Disease Control and Prevention and the American College of Sports Medicine. JAMA 273: $402-407$.

22. Haskell WL, Lee IM, Pate RR, Powell KE, Blair SN, et al. (2007) Physical activity and public health: updated recommendation for adults from the American College of Sports Medicine and the American Heart Association. Circulation 116: 1081-1093.

23. Friedewald WT, Levy RI, Fredrickson DS (1972) Estimation of the concentration of low-density lipoprotein cholesterol in plasma, without use of the preparative ultracentrifuge. Clin Chem 18: 499-502.

24. Bucolo G, David H (1973) Quantitative determination of serum triglycerides by the use of enzymes. Clin Chem 19: 476-482.

25. Trinder $P$ (1969) Determination of blood glucose using an oxidase-peroxidase system with a non-carcinogenic chromogen. J Clin Pathol 22: 158-161.

26. Gillespie CD, Keenan NL, Miner JB, Hong Y; Centers for Disease Control and Prevention (CDC) (2012) Screening for lipid disorders among adults--National Health and Nutrition Examination Survey, United States, 2005-2008. MMWR Morb Mortal Wkly Rep 61 Suppl: 26-31.

27. Alencastro PR, Wolff FH, Ikeda ML, Schuelter-Trevisol F, Brandão ABM, et al. (2012) Diagnostic criteria for lipodystrophy in HIV-infected patients (submitted).

28. Tien PC, Cole SR, Williams CM, Li R, Justman JE, et al. (2003) Incidence of lipoatrophy and lipohypertrophy in the women's interagency HIV study. J Acquir Immune Defic Syndr 34: 461-466.

29. Alberti KG, Zimmet P, Shaw J; IDF Epidemiology Task Force Consensus Group (2005) The metabolic syndrome--a new worldwide definition. Lancet 366: 10591062.

30. NHANESIII. National Health and Nutritional Examination Survey III: Body Measurements

31. Lichtenstein KA, Ward DJ, Moorman AC, Delaney KM, Young B, et al. (2001) Clinical assessment of HIV-associated lipodystrophy in an ambulatory population. AIDS 15: 1389-1398.

32. Glesby M, Desai A, Erbelding E, Kostman J (1999) High prevalence of impaired glucose tolerance prior to initiating protease inhibitor therapy. In: Sixth Conference on Retroviruses and Opportunistic Infections, Chicago.

33. Saint-Marc T, Partisani M, Poizot-Martin I, Bruno F, Rouviere O, et al. (1999) A syndrome of peripheral fat wasting (lipodystrophy) in patients receiving longterm nucleoside analogue therapy. AIDS 13: 1659-1667.

34. Grunfeld C (2008) Understanding the complications of antiretroviral drugs. Clin Infect Dis 47: $575-576$

35. Martinez E, Mocroft A, García-Viejo MA, Pérez-Cuevas JB, Blanco JL, et al (2001) Risk of lipodystrophy in HIV-1-infected patients treated with protease inhibitors: a prospective cohort study. Lancet 357: 592-598.

36. Jacobson DL, Tang AM, Spiegelman D, Thomas AM, Skinner S, et al. (2006) Incidence of metabolic syndrome in a cohort of HIV-infected adults and prevalence relative to the US population (National Health and Nutrition Examination Survey). J Acquir Immune Defic Syndr 43: 458-466.

37. Lichtenstein KA, Delaney KM, Armon C, Ward DJ, Moorman AC, et al. (2003) Incidence of and risk factors for lipoatrophy (abnormal fat loss) in ambulatory HIV-1-infected patients. J Acquir Immune Defic Syndr 32: 48-56.

38. Lichtenstein KA (2005) Redefining lipodystrophy syndrome: risks and impact on clinical decision making. J Acquir Immune Defic Syndr 39: 395-400.

39. Alencastro PR, Wollf FH, Oliveira RR, Ikeda ML, Barcellos NT, et al. (2012) Metabolic syndrome and population attributable risk among HIVIAIDS patients: comparison between NCEP-ATPIII, IDF and AHA/NHLBI definitions. AIDS Res Ther 9: 29.

40. Friis-Møller N, Weber R, Reiss P, Thiébaut R, Kirk O, et al. (2003) Cardiovascular disease risk factors in HIV patients--association with antiretroviral therapy. Results from the DAD study. AIDS 17: 1179-1193.

41. Schuelter-Trevisol F, Alencastro PR, Ribeiro PAB, Wolff FH, Ikeda MLR, et al. (2012) Association of Physical Activity with Lipodystrophy Syndrome in HIVInfected Patients. J AIDS Clinic Res 3:177. 
Citation: Alencastro PR, Wolff FH, Schuelter-Trevisol F, Ikeda ML, et al. (2012) Characteristics Associated to Lipodystrophy Syndrome among HIVInfected Patients Naïve and on Antiretroviral Treatment. J AIDS Clinic Res 3:182. doi:10.4172/2155-6113.1000182

Page 9 of 9

42. Brown TT, Li X, Cole SR, Kingsley LA, Palella FJ, et al. (2005) Cumulative exposure to nucleoside analogue reverse transcriptase inhibitors is associated with insulin resistance markers in the Multicenter AIDS Cohort Study. AIDS 19: 1375-1383.
43. Kosmiski LA, Kuritzkes DR, Lichtenstein KA, Glueck DH, Gourley PJ, et al. (2001) Fat distribution and metabolic changes are strongly correlated and energy expenditure is increased in the HIV lipodystrophy syndrome. AIDS 15: 1993-2000. 